\title{
The Physical and Optical Societies' Exhibition.
}

THE sixteenth annual Exhibition of Electrical, Optical, and other Physical Apparatus held by the Physical Society of London and the Optical Society on January 5,6 , and 7 , was marked by a new departure which added greatly to its interest and popularity. In addition to the trade display of scientific apparatus, a number of demonstrations were provided in illustration of typical results of modern research and new or improved laboratory methods, together with lecture experiments and repetitions of famous historical experiments. After the admission of ticket-holders for the usual two days, the Exhibition was thrown open to the general public for a further day, and the available accommodation at the Imperial College of Science and Technology, South Kensington, was fully occupied throughout. There can be no doubt as to the success of the new section, and it is to be hoped that in future years it may be greatly extended. It makes considerable demands on the time of those who take part in it, as the experiments have to be carefully prepared, but public spirit of this kind has never been lacking amongst physicists. Prof. A. O. Rankine must be congratulated on the feat of organisation which he achieved, and mention must be made of the excellent illustrated catalogue, containing brief descriptions of the more important exhibits, which is now printed in a form suitable for inclusion in the bound volumes of the Proceedings of the two Sócieties.

In the historical section, pride of place must be given to a repetition of some of Newton's fundamental experiments in optics, prepared by Prof. F. J. Cheshire and Mr. B. K. Johnston. Elaborate care was taken to reproduce the original conditions as closely as possible, and an artificial sun was employed, comprising a source of light to which the required angular diameter was imparted by means of a lens system. Sir William Bragg illustrated Faraday's discovery of electromagnetic induction with apparatus which included the coil actually used by Faraday, and Mr. F. E. Smith showed the British Association rotating coil with which Maxwell measured resistance in primary units.

Amongst the more striking lecture experiments may be mentioned those by Mr. G. L. Addenbrooke on electrostatic attraction and by Dr. L. C. Martin on the polarisation of light. In the former, one sparking-ball of a Wimshurst machine is located over a saucerful of castor oil, a few millimetres above the surface. When the machine is operated, a column of oil rises up and adheres to the ball so long as the latter remains charged. In Dr. Martin's experiment, a beam of plane-polarised light is reflected vertically downwards through a sugar solution clouded by particles in suspension. Since the scattered light is seen with maximum intensity in the plane in which it is polarised, and this plane is rotated by the solution at different rates for different colours, the colour of the beam appears to change along its length. A current weigher designed by Mr. F. E. Smith should be of value in getting students interested in the subject of absolute measurements. It comprises a large fixed vertical coil, and a small central horizontal coil which swings about the horizontal diameter of the former. The current to be measured is passed through the two coils in series and the resulting torque is balanced by weights added to a scale pan, which is attached to the moving coil at a suitable distance from its axis.

The research section comprised exhibits by the more important research departments maintained by the Government and by industrial firms. An interesting item in the Admiralty's contribution was the piezo-electric oscillograph. A quartz strip with metal faces contracts longitudinally when the faces are oppositely charged, and this movement may be applied directly to an optical lever; or two strips of quartz may be cemented together in piezo-electric opposition so as to give a bending movement like that of the bimetallic strips used in thermostats. The National Physical Laboratory's exhibits included a Ewing flowmeter (Dr. E. Griffiths and Mr. J. H. Awbery), in which water flowing upwards in a tube containing a steel ball keeps the ball at a height which depends on the rate of flow. When the tube is vertical the ball may either rotate steadily or oscillate from side to side of the tube : two markedly different calibrations are applicable to these two alternative states. Mr. J. Guild showed a colorimeter which enables any mixed colour to be plotted on the colour triangle by means of purely qualitative measurements. The colour is matched against a mixture of a pure red of known wave-length from a filter with monochromatic light selected from a spectrum, the intensities being varied as required but not measured. A similar observation is made with a blue instead of a red filter, and the two wavelengths of the colours selected from the spectrum determine the required co-ordinates. A useful laboratory method for splitting mica was shown by Mr. F. Harrison Glew. Two pieces of adhesive tape are attached to the faces of a mica sheet and pulled apart. This operation starts a split which can be extended with a piece of cardboard, and by repeating the process a sufficient number of times, sheets giving interference colours can be obtained without difficulty.

A discourse was delivered each evening during the Exhibition. At the first, on "The Search for Ultra-Microscopic Organisms," by Mr. J. E. Barnard, the attendance was so great that a number of persons were unable to obtain admission, but the speaker's good nature and endurance proved equal to an immediate repetition of the discourse for their benefit. Mr. Barnard explained that the resolution obtainable with a microscope is limited on one hand by the formation of diffraction patterns if the wave-length be too long, and on the other by the opacity of the lens-material if the wave-length be too short. The organisms which are to be photographed have to be grown on a nutritive layer as thin as $0 \cdot 2 \mu$, in order to avoid opacity, and the wave-length varies from $220 \mu \mu$ upwards, different wave-lengths giving different pictures, owing to differential absorption in the tissues of the object. The microscope combines two lens systems which are appropriate respectively to visible and to ultra-violet radiation, have one quartz lens in common, and are focussed by means of the same mechanism. The latter is calibrated, so that when focussing has been accomplished for visible light of known wave-length, the instrument can at once be set, with the aid of the calibration, for the required ultra-violet illumination. The visible light is used with dark-ground illumination, the ultra-violet with a camera. The organisms of malignant growths are found to be so small that the ratio of their surface tension to their mass is too great to permit of reproduction by central fission, and young individuals make their appearance as a series of thickenings in the cell wall of the parent, which ultimately remain attached thereto only by a spiral filament.

Prof. A. F. C. Pollard's discourse dealt with " The Mechanical Design of Instruments." $\mathrm{He}$ claimed that a scientific instrument must be looked upon

$$
\text { NO. 2933, vOL. II } 7]
$$


as a mechanism, for transmitting motion rather than power, and that it must be designed in strict accordance with kinematic principles. The design of a pair or coupling is governed by the principle that a part is fixed in position when its six possible degrees of freedom have all been constrained by the location of six of its points upon six planes. Any further constraint, such as exists in most instruments, is redundant and involves the accurate fitting together of parts, which is far more difficult and costly than the accurate geometrical shaping of parts taken by themselves: the latter process only is required when redundant constraints are avoided, and at the same time the effects of wear due to imperfect fit are eliminated. The principle was illustrated by reference to actual instruments in which such mechanisms as the hole, slot, and plane of Lord Kelvin and the rectangular knife-edge arrangement of Sir Horace Darwin have been employed. As regards the linlss or rigid parts of an instrument, their design must be governed by a knowledge of what is practicable in a workshop: for example, castings must not have thin parts integral with adjacent thick parts, and holes must not be located so that in drilling them the tool has to work through a face to which it is inclined.

The discourse by Major W. S. Tucker on " Electrical Listening" was illustrated by a number of demonstrations of the properties of microphones, particularly of the tuned microphones associated with his name and used in military sound-ranging. These instruments; in which a very fine hot wire forming one arm of a Wheatstone bridge is arranged as a mesh across the mouth of a Helmholtz resonator, have been given a much enhanced selective sensibility for low frequency disturbances by closely coupling the main resonator with a second resonator of equal pitch. Perhaps the most striking experiment was that in which the speed of a siren was gradually decreased. The resonant microphone in combination with a vibration galvanometer gave a strongly marked indication as each harmonic of the siren passed through the selected pitch. The speaker also showed records of the notes emitted by lightships, taken at a distance of 9 miles, and of thunderclaps, the indicated duration of the latter being much longer than the duration apparent to the ear.

The trade exhibits included a large number of beautiful instruments, many of them new or improved, but space is available for the description of only very few of these. A fuller account has been published in the illustrated catalogue of the Exhibition. The attractive exhibit of Messrs. Adam Hilger, Ltd., included a demonstration with one of their piezoelectric resonators, which are used in standardising wireless wave-lengths. The crystal employed has a mechanical resonance frequency comparable with wireless frequencies, and when it is connected in a suitable circuit, the marked oscillatory piezo-electric E.M.F. which is set up at the fundamental and harmonics of the mechanical resonance enables it to be used for standardising wave-meters, the minimum wave-length being 20 metres. The most interesting feature of the stand occupied by the Cambridge Instrument Co., Ltd., was an improved design of Duddell oscillograph. This instrument is now fully portable, gives direct vision and three simultaneous records on $50 \mathrm{~cm}$. or shorter strips of ordinary photographic paper, permits of any combination of electromagnetic and electrostatic vibrators and employs a filament lamp. A novelty in dial recorders is the use of carbon paper by the Foster Instrument Co., the stylus making its impression from behind so as to press the carbon paper upon the semi-transparent record paper against the glass face of the instrument. A new method for preventing the bursting, under excess pressures, of the expanding metal chambers used in barometers and the like is embodied in a thermostat by Negretti and Zambra: in this case the chamber is enclosed in a gas-tight chest and the pressure acts upon it from without. Several ingenious features are also to be found in the Ashdown rotoscope (Elliott Brothers (London), Ltd.) for observing periodic movements such as those of machine parts or even of a flying insect. The stroboscopic shutter is a rotating disc driven by clockwork through a fivespeed gear, intermediate speeds being adjusted by means of a governor control. The total range of speeds is from 300 to 20,000 r.p.m. The motor can be started or stopped by turning or holding its shaft with the hand, the difference between static and dynamic friction being utilised to this end in the design; the instrument can be held in one hand when in use.

Much interest was shown in a working example of the Admiralty's apparatus for sounding depths by timing echoes, which is being manufactured by Messrs. Henry Hughes and Son, Ltd. The principle employed is that in which a signal note is emitted at regular intervals and a normally deaf telephone is momentarily rendered sensitive after the lapse of an adjustable interval. The length of this interval when the adjustment is such that the echo can be caught by the telephone measures the depth of the sea. It is believed that the use of a rapidly damped signal note, in combination with other detail improvements, has yielded a fool-proof apparatus. Messrs. C. F. Casella and Co., Ltd., exhibited a surveying instrument designed by Dr. J. S. Owens, for recording the inclination of a bore hole in elevation and azimuth. The instrument contains a complete photographic chronograph, a magnetic compass mounted in gimbals, and a clinometer, and it can be inserted complete into a 2 -inch bore hole. It should serve to prevent the distress felt by engineers when a borer, after much apparently successful labour, is found to have behaved like a boomerang. Telephones for the relief of deaf persons have been in use for a long time, but have often proved unsatisfactory owing to the variety in types of deafness. To overcome this difficulty Mr. W. H. Pettifor has designed a test instrument which enables prescriptions to be written for individual patients, analogous to opticians' prescriptions; it is found that each patient requires a combination of microphone and telephone having a particular frequency-characteristic and sensitivity.

In all, some seventy-two firms exhibited. It is, naturally, impossible in the space available in NATURE to describe more than a few of the many important instruments which went to make up this impressive display.

\section{Regional Scientific Work.}

THOSE interested in the publications of the local scientific societies in Great Britain will have noticed that after the falling off in the printing of memoirs which occurred during the War, there has been a healthy revival. Notwithstanding the enormous increase in the cost of printing and binding, many of the scientific societies have reached the pre-War standard of their reports, and in some cases have even exceeded that. A perusal of the enormous number of reports and transactions recently issued shows that much more discrimination is now being taken in the publication of papers by the scientific 